\title{
LASER INFRAROUGE À BASE DE SEMI-CONDUCTEURS DE LA FILIĖRE SILICIUM
}

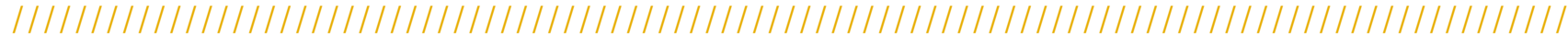

Moustafa EL KURDI ${ }^{*}$, Emilie SAKAT ${ }^{1}$, Jean-Michel HARTMANN ${ }^{2}$, Vincent REBOUD ${ }^{2}$,

Alexei CHELNOKOV ${ }^{2}$, Nicolas PAUC $^{3}$, Vincent CALVO ${ }^{3}$, Philippe BOUCAUD ${ }^{4}$, Fréderic BCUF ${ }^{5}$

${ }^{1} \mathrm{C} 2 \mathrm{~N}$-Université Paris-Saclay- CNRS, Palaiseau, France

${ }^{2}$ CEA, LETI, Université Grenoble Alpes, Grenoble, France

${ }^{3}$ CEA, IRIG-DePhy, Université Grenoble Alpes, Grenoble, France

${ }^{4}$ CRHEA, Université Côte d'Azur- CNRS, Sophia-Antipolis, France

${ }^{5}$ STMicroelectronics, Crolles, France

*moustafa.el-kurdi@c2n.upsaclay.fr

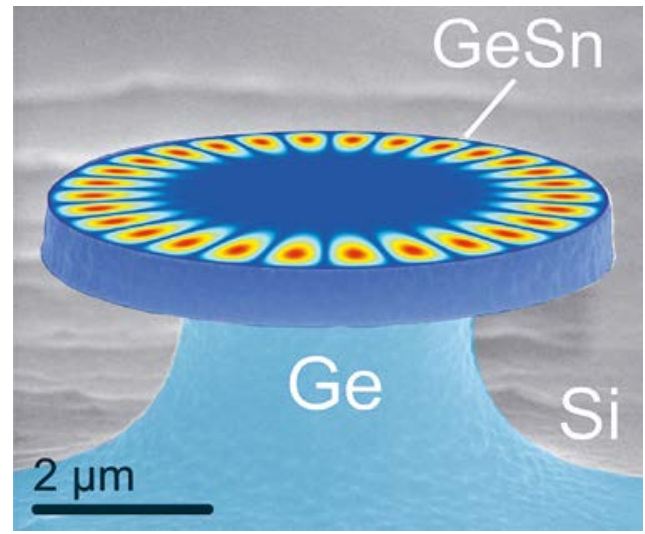

Nous présentons les récents développements scientifiques et techniques liés aux sources lasers infrarouges en micro-cavités à base d'alliages germanium-étain (GeSn). Ces alliages sont des matériaux semi-conducteurs de la filière silicium compatibles avec les procédés de fabrication bas coût de l'industrie de la micro-électronique. Un des enjeux est d'obtenir un alignement direct de la structure de bande électronique avec ces éléments de la colonne IV.

https://doi.org/10.1051/photon/202110940

Article publié en accès libre sous les conditions définies par la licence Creative Commons Attribution License CC-BY (https://creativecommons.org/licenses/by/4.0), qui autorise sans restrictions l'utilisation, la diffusion, et la reproduction sur quelque support que ce soit, sous réserve de citation correcte de la publication originale.

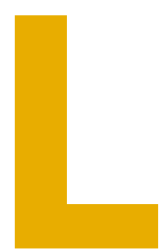

es technologies photoniques basées sur le silicium et compatibles CMOs (Complementary Metal Oxide Semiconductor) ont permis des avancées significatives dans le traitement optique sur puce, en particulier pour la détection et la modulation rapides de signaux optiques dans la gamme de longueurs d'onde du proche infrarouge (IR). L'un des principaux inconvénients de cette filière est en revanche le manque de sources lasers compatible avec les technologies CMOS. En effet, les éléments de la colonne IV compatibles avec le silicium (germanium, alliages SiGe...) sont des semi-conducteurs à bande interdite indirecte ce qui les a toujours empêchés de générer du gain optique et d'être utilisés dans des technologies laser. Pour pallier ce manque et stimuler les technologies photoniques silicium, des efforts significatifs ont été consacrés à l'intégration de lasers hautes performances à base de composés III-V, sur plateforme photonique SOI (silicon-on-insulator) notamment. Cela a été particulièrement vrai pour les applications datacom proche infrarouge, aux longueurs d'onde utilisées pour la transmission par fibre optique. Les lasers issus de la filière III-V sont les sources de lumière les plus standards et les plus fiables qui existent actuellement et peuvent être reportés sur silicium suivant différentes techniques, comme 
a
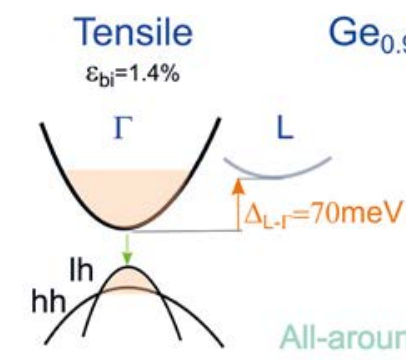

All-around stressor

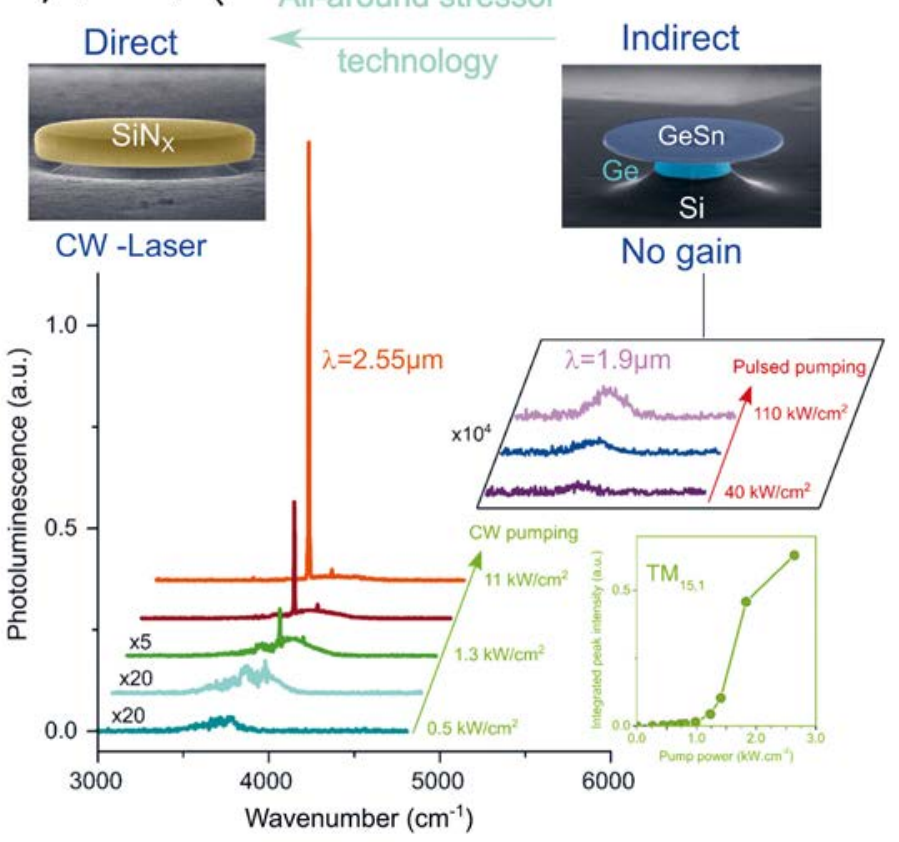

b
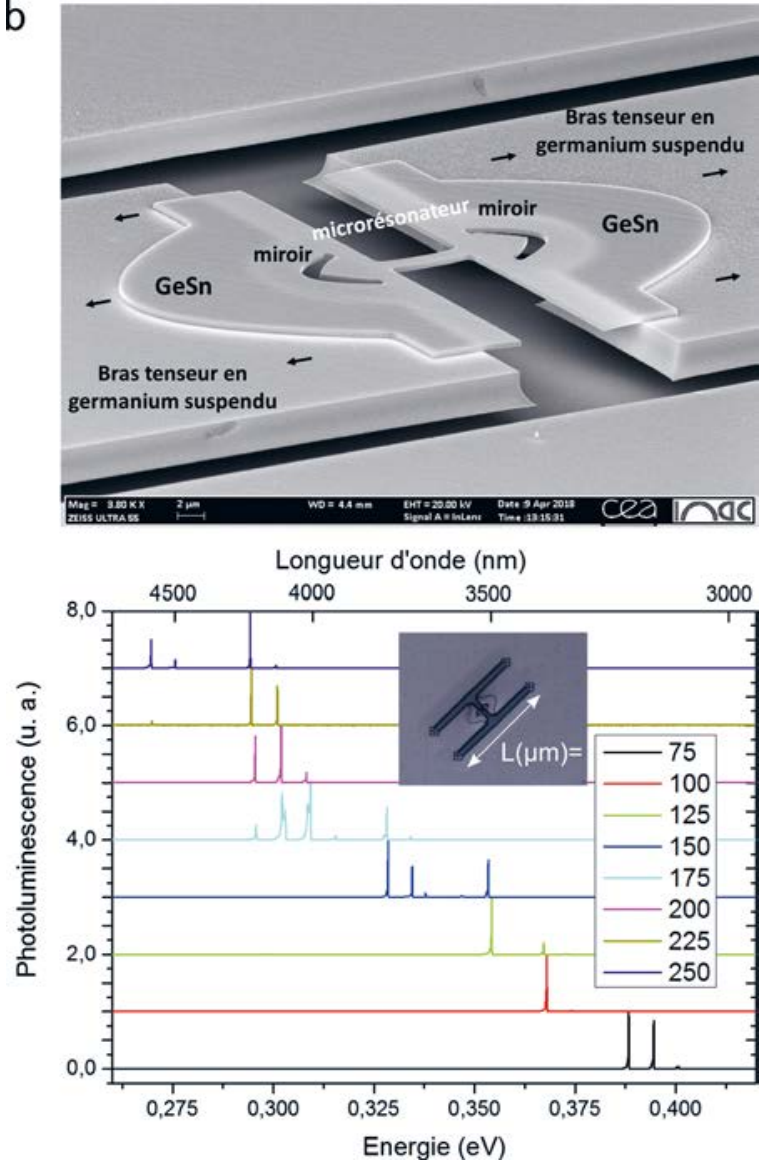

Figure 1.a. Première approche : schéma de la structure de bande d'un alliage GeSn avec $5.4 \%$ de Sn. En fabriquant un microdisque à partir de cet alliage épitaxié sur substrat virtuel de Ge sur silicium, la cavité optique présente une déformation résiduelle proche de $0 \%$ mais ne permet pas de montrer un effet laser, le matériau étant à bande indirect. En lui appliquant une déformation en tension, suivant la technologie all-around sur un microdisque, l'alliage présente une bande interdite directe avec une directivité $\Delta_{\mathrm{L}-\mathrm{r}}$ de $70 \mathrm{meV}$. Cette approche permet d'obtenir un effet laser en pompage continu avec un seuil de l'ordre de $1 \mathrm{~kW} / \mathrm{cm}^{2}$. Le disque contraint en tension présente une émission fortement décalée vers le rouge, de $1.9 \mu \mathrm{m}$ à $2.5 \mu \mathrm{m}$ de longueur d'onde, en raison de la déformation biaxiale appliquée de $1.4 \%$. b. La deuxième approche consiste à structurer la couche de GeSn en micro-ponts maintenus par des bras de Ge suspendus. Les bras de Ge étant légèrement en tension celle-ci est exaltée au niveau du pont de GeSn permettant ainsi de lui appliquer une déformation variable en fonction de la longueur des bras. Deux miroirs de type coin cube permettent de former une cavité FabryPérot. Ce dispositif réalisé à partir d'une couche de GeSn avec $16 \%$ d'étain permet d’obtenir une émission laser accordable sur une plage de 1.5 m de longueur d'onde faisant varier la longueur des bras. L'effet laser est obtenue dans ce cas jusqu'à $270{ }^{\circ} \mathrm{K}$, sachant que la plus forte composition d'étain permet d'obtenir une plus forte directivité $\Delta_{\mathrm{L}-\mathrm{r}}$.

l'épitaxie directe et via des couches tampon (couches intermédiaires d'adaptation de paramètre de maille ou encore appelées substrats virtuels), ou encore en utilisant des procédés de collage, on parle alors d'intégration hétérogène de III-V sur Si. Le coût de fabrication de ce type de procédé s'avère néanmoins très élevé, et l'incompatibilité chimique des III-V avec les éléments IV rend leur intégration sur les lignes de fabrication CMOS encore plus complexe.
Plus récemment, il a été découvert quel'on peut obtenir une bande interdite directe, favorable à l'obtention de gain optique, avec des alliages GeSn à condition d'ajuster correctement leur composition et leur déformation en tension. Typiquement, pour des teneurs en étain supérieures à 7-8 \% un alignement direct des bandes est obtenu même si l'alliage est relaxé mécaniquement. En revanche, pour des teneurs inférieures à $7 \%$, il est nécessaire d'appliquer une déformation en tension. Pour donner un ordre de grandeur, une tension au-delà de $2 \%$ en biaxial ou de $5.5 \%$ en uniaxial est nécessaire dans le cas du germanium pur.

Cette stratégie reposant sur l'insertion d'atomes d'étain en substitution dans un cristal de germanium conduit par ailleurs à une bande interdite directe réduite par rapport à celle du germanium pur. Ces alliages sont ainsi adaptés à l'émission ou la détection de lumière dans la gamme [2-5] $\mu \mathrm{m} \bullet \bullet \bullet$ 
del'IR, gamme de longueur d'onde assez peu couverte par les lasers de la filière III-V. Or de nombreuses molécules, dont celles des gaz à effets de serre, ont des signatures d'absorption dans cette gamme de longueur d'onde. Ainsi en plus de leur technologie bas coût, les lasers GeSn ouvrent de nouveaux champs d'application tels que la détection biochimique et le contrôle de l'air.

\section{LASER GESN : INGÉNIERIE DE CONTRAINTE DANS DES MICRORÉSONATEURS}

Les recherches récentes sur ce nouveau type de laser à base d'alliage GeSn se sont donc particulièrement portées sur l'ingénierie de la structure de bande électronique (composition et déformation en tension) en vue d'obtenir un alignement direct nécessaire à la naissance du gain optique. La première difficulté pratique a résidé dans l'obtention d'une composition détain suffisante (supérieure à 7-8 \% notamment), sachant que la solubilité limite du Sn dans le Ge à l'équilibre n'est que de $1 \%$. De nombreux efforts se sont ainsi portés sur le développement de procédés de croissance métastables afin de parvenir à incorporer plus d'étain. À l'heure actuelle, il est possible d'obtenir des compositions jusquà $16 \%$ en utilisant des techniques CVD (Chemical Vapour Deposition). La deuxième difficulté est de gérer le désaccord de maille entre le GeSn et le silicium. L'utilisation d'une couche tampon de Ge pur déposée au préalable sur le substrat de silicium permet de remédier en partie à ce problème. Un désaccord de

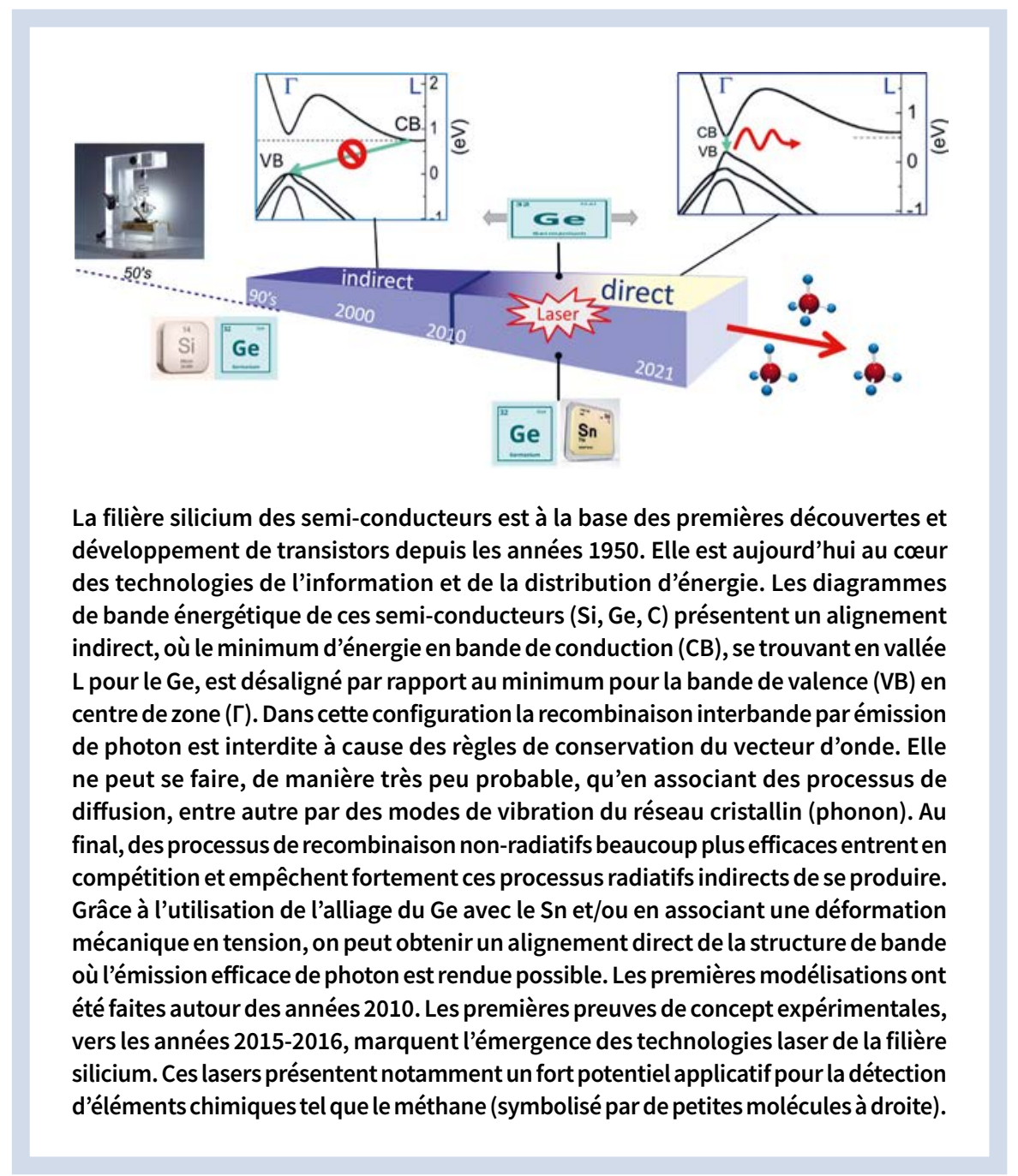

maille demeure néanmoins entre le $\mathrm{GeSn}$ et le Ge, à mesure notamment que la composition d'étain augmente. Il en résulte des problématiques de contrainte en compression rédhibitoire car la couche fine (inférieure à lépaisseur critique avant relaxation) de GeSn épitaxiée se retrouve dans un état qui induit une réduction de la directivité de la bande interdite, voire même la rend indirecte. Pour contourner ce problème, l'approche usuellement adoptée est de faire relaxer cette contrainte en faisant croître des épaisseurs supérieures aux épaisseurs critiques de relaxation plastique, mais ce procédé induit malheureusement la formation d'un réseau dense de dislocations dans le GeSn, près de l'interface avec la couche tampon de Ge qui sont largement préjudiciables à la naissance de l'effet laser. En conséquence les premières démonstrations de laser à base de GeSn utilisaient des densités de puissance seuil de l'ordre de la centaine de $\mathrm{kW} / \mathrm{cm}^{2}$ en régime impulsionnel et fonctionnaient à des températures cryogéniques [1]. L'ingénierie de contrainte et de défauts dans le GeSn présentent donc des enjeux technologiques importants.

En appliquant une déformation en tension, on peut réduire la quantité de $\mathrm{Sn}$ nécessaire à l'obtention d'un gap direct et en même temps réduire les difficultés liées aux défauts de désaccord de maille. Il faut néanmoins appliquer des niveaux de déformation assez extrêmes à mesure qu'on se rapproche du germanium pur [2]. Il est donc nécessaire de développer des stratégies expérimentales et des schémas de structures photoniques tout à fait spécifiques afin d'explorer les différents paramètres influant le gain optique. Il est en particulier crucial de s'appuyer sur des structures permettant d'appliquer des déformations en tension importante.

Notre équipe a en ce sens récemment proposé une technologie allaround où un film stressor de $\mathrm{SiNx}$ entoure complètement un micro-résonateur de GeSn (voir Fig. 1-a). La distribution de déformation obtenue 
est très homogène dans la microcavité en GeSn [3] et un niveau record de déformation en tension dans une cavité résonnante de type microdisque, à hauteur de $1.4 \%$ biaxiale, a été atteint. De plus, la technologie proposée repose sur un report de la couche de GeSn par une technique de collage et retrait de substrat, ce qui permet de retirer la zone de défaut d'accord de maille dans le GeSn (cette dernière se retrouvant tout au-dessus) et d'améliorer sa qualité structurale. Cette approche a été appliquée sur une couche avec une teneur en étain de seulement $5.4 \%$, donc initialement à bande interdite indirecte et ne pouvant pas générer de gain optique. L'alignement de la structure de bande obtenue est direct, avec une directivité $\Delta_{\mathrm{L}-\Gamma}$ de $70 \mathrm{meV}$ permettant d'atteindre un régime laser (Fig. 1-a). Par ailleurs, la forte déformation appliquée induit une levée de dégénérescence de la bande de valence, donc une réduction de la densité d'états et contribue, avec le retrait des dislocations, à réduire les seuils d'inversion de population. En suivant cette approche, l'effet laser a été obtenu pour la première fois en régime continu avec des densités de seuils de l'ordre de $1 \mathrm{~kW} / \mathrm{cm}^{2}$, soit réduits de 2 ordres de grandeur par rapport à l'état de l'art [4]. Dans ce cas, les performances sont très similaires à celles que l'on peut avoir avec des lasers III-V reportés sur silicium, à la différence que l'oscillation laser n'est maintenue que jusqu'à $100^{\circ} \mathrm{K}$ alors que les lasers III-V fonctionnent à température ambiante. L'idéal serait d'augmenter la directivité qui se caractérise par la barrière d'énergie entre les états d'électron en $\Gamma$ et les états en vallée $L$ (nommé $\Delta_{\text {L-r }}$ sur la Fig. 1). Ainsi la population d'électrons serait mieux maintenue dans la vallée directe $(\Gamma)$, et le gain optique pourrait être conservé lorsque la température augmente.

Une deuxième approche consiste à utiliser des alliages à plus forte composition d'étain, typiquement de $16 \%$, qui présentent une plus grande directivité, de l'ordre de $150 \mathrm{meV}$, en fonction de la déformation appliquée. Récemment cette approche, associée à une ingénierie de contrainte en structure micro-ponts (Fig. 1-b), a permis d'atteindre un régime laser jusqu'à $270{ }^{\circ} \mathrm{K}$ avec de plus la possibilité d'accorder la longueur d'onde d'émission via les paramètres géométrique du dispositif [5]. Les seuils laser sont néanmoins de l'ordre du $\mathrm{MW} / \mathrm{cm}^{2}$ lorsqu'on s'approche de l'ambiante.

\section{CONCLUSION}

L'alliage du Ge avec l'étain associé à de l'ingénierie de contrainte et à la gestion des défauts devrait conduire au chainon manquant de la filière silicium : une source laser sous injection électrique et à température ambiante. Cet article a permis de présenter quelques éléments essentiels autour des technologies émergentes et très prometteuses qui ont déjà permis d'obtenir des premières démonstrations sous pompage optique et des seuils très bas. De futures données expérimentales devraient permettre de décrire plus finement l'influence des différents paramètres sur la robustesse $d u$ gain optique, et ainsi conduire à des designs optimaux. Il est à noter que d'autres voies sont en cours de développement en vue de réaliser des lasers encore plus performants : le potentiel d'hétérostructures de types puits quantiques et boîtes quantiques comme milieu à gain fait notamment l'objet d'explorations diverses.
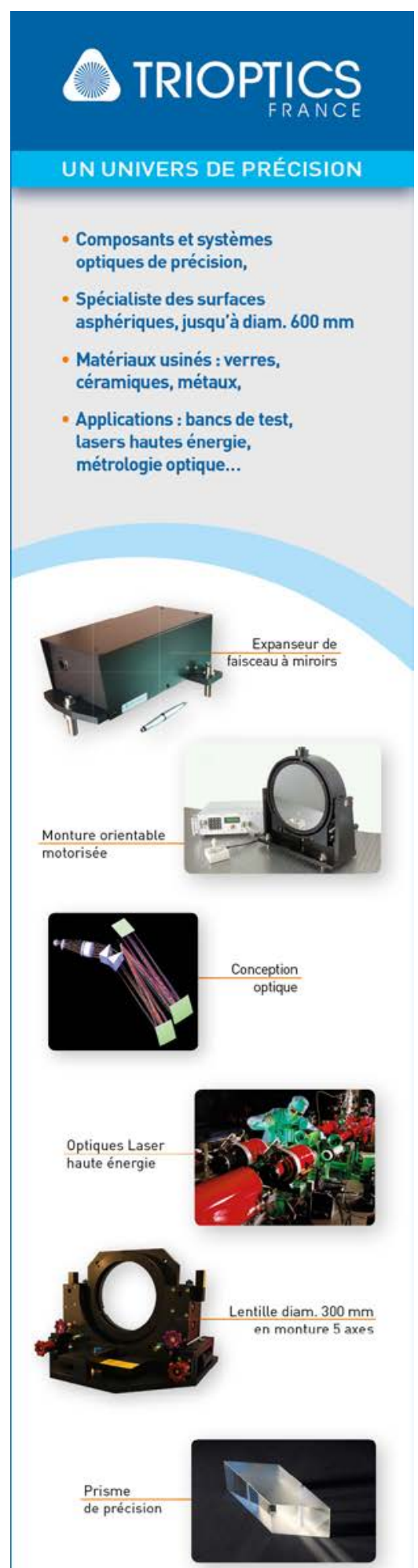

Optical Surfaces ( 3

Trioptics France

76 rue d'Alsace

69100 Villeurbanne

Téll. 0772440203

Fax: 0472440506

www.trioptics.ff 


\title{
Principes essentiels de conception et de spécification des optiques laser
}

\author{
Les ingénieurs exploitent de nouveaux matériaux, des mécanismes de gain inhabituels et des \\ conceptions de cavités innovantes pour pousser les performances du laser vers de nouveaux \\ horizons. Les durées d'impulsion sont de plus en plus courtes, le pic de puissance est de plus en \\ plus important et l'énergie des photons est de plus en plus élevée à mesure que les systèmes \\ passent à des longueurs d'onde ultraviolettes (UV) courtes.
}

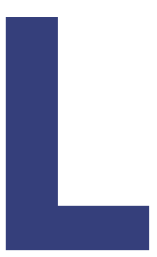

es concepteurs de systèmes laser doivent veiller à utiliser des optiques conçues pour s'adapter à la puissance de crête et à la densité d'énergie de leur source laser. Les longueurs d'onde UV sont plus énergétiques que leurs homologues visibles ou infrarouges, et les préoccupations deviennent donc plus importantes pour les optiques des lasers UV.

La rugosité et la qualité de surface sont particulièrement importantes pour les surfaces optiques intra- et extra-cavité. Toute énergie absorbée par les imperfections de l'optique peut entraîner un échauffement rapide, ce qui dégrade davantage cette dernière et peut conduire à la défaillance des composants. De plus, avec les systèmes laser à haute énergie, il peut y avoir de graves conséquences lorsqu'un élément diffuse la lumière là où elle ne devrait pas se trouver. La réduction de cette diffusion conduit également à des systèmes à faibles pertes et à haut débit, ce qui est essentiel pour de nombreuses applications laser. Lorsqu'ils évaluent les fournisseurs de composants, les concepteurs ne doivent pas se limiter aux spécifications annoncées ; ils doivent également s'assurer que leur fournisseur peut mesurer les caractéristiques qu'il prétend produire.

\section{EN DOUCEUR, TOUT EN DOUCEUR}

Une grande partie du travail de tout système optique se fait sur les surfaces; une courbure correcte est donc essentielle. Une lentille ou un miroir qui ne présente aucun écart par rapport à sa courbure de conception se comporte exactement comme prévu, mais est également impossible à créer. Il y aura toujours des déviations par rapport à la perfection lors de la fabrication. Les écarts de basse fréquence spatiale par rapport à la forme idéale sont classés comme des erreurs de figure de surface. Ces erreurs réduisent les performances d'un système optique en décalant la propagation du faisceau, en déviant le trajet de certaines de ses parties. Cela peut rendre les images floues ou éloigner des parties d'un faisceau de sa cible.

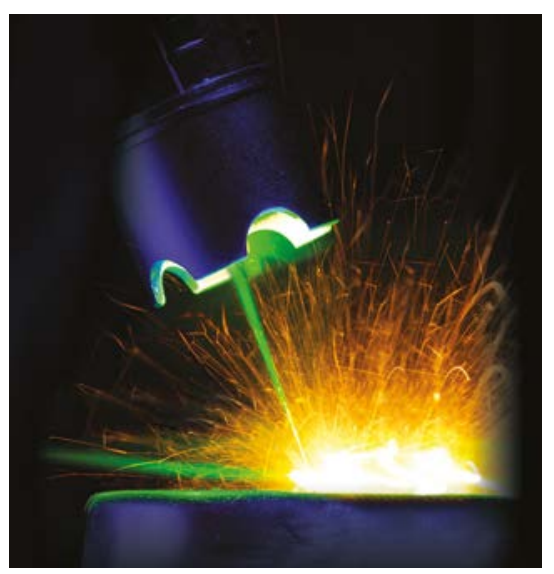

Figure 1. Les optiques laser nécessitent des tolérances de surface plus strictes que les composants optiques standard afin de maintenir les performances dans des applications telles que le traitement des matériaux.

Les déviations à des fréquences spatiales plus élevées créent une classe différente de dégradation des performances et sont communément appelées « erreurs de finition de surface ». Un type d'erreur de finition de surface est appelé « rugosité de surface», qui est une mesure de la variation d'un profil de surface par rapport à son profil idéal mesuré sur une gamme donnée de hautes fréquences spatiales. La rugosité de surface admissible est spécifiée par la divergence maximale du profil de surface à une fréquence spatiale donnée ou une plage de fréquences spatiales. L'effet principal de la rugosité de surface est la diffusion de la lumière, qui envoie des parties d'un faisceau lumineux à des angles élevés par rapport au faisceau primaire de propagation. La diffusion peut être un problème avec tout système optique, car elle réduit la transmission effective et peut même finir par créer des signaux parasites au niveau du détecteur du système. Dans les systèmes laser, la lumière diffusée peut être encore plus préjudiciable car elle peut transporter des quantités importantes d'énergie, ce qui peut endommager les composants d'un système et même présenter un risque pour la sécurité.

Un autre type d'erreur de finition de surface se présente sous la forme de défauts ponctuels. Ces types d'erreurs sont souvent classés comme des « rayures » et des "creux», et sont couverts par les normes sur les rayures et les creux (Scratch-Dig), qui définissent à la fois la taille et le nombre autorisés de ces défauts esthétiques. Les défauts de type «Scratch-Dig » diffusent également la lumière, dégradant les performances d'un système de la même manière que la rugosité de surface. Les dommages laser commencent souvent par ce type de défauts.

Presque toutes les optiques laser sont traitées, de sorte que (bien que la qualité du substrat ne soit pas sans importance) la surface de l'optique est définie par la surface du traitement. Les optiques laser sont généralement conçues pour être utilisées à une seule longueur d'onde ou à un ensemble de longueurs d'onde harmoniques, de sorte que les traitements sont souvent conçus pour réfléchir ou transmettre un pourcentage élevé de ces longueurs d'onde cibles. La durabilité du revêtement estégalement importante, en 
particulier dans des conditions de forte humidité ou de forte variation de température.

En général, la diffusion est plus importante au niveau des longueurs d'onde courtes. C'est pourquoi les optiques laser à courte longueur d'onde sont les plus affectées par la rugosité et la qualité de surface. Il incombe aux ingénieurs des systèmes laser UV de déterminer avec soin les spécifications relatives à la rugosité de surface et aux rayures et creux. Toutefois, la qualité d'une spécification n'a pas d'importance si le fournisseur ne peut pas la vérifier à l'aide de ses outils métrologiques. Les concepteurs de systèmes optiques doivent vérifier que leur fournisseur utilise la bonne métrologie pour mesurer réellement les performances qu'ils prétendent atteindre.

La microscopie à force atomique (AFM) est un outil précieux pour mesurer la finition de la surface, caractériser la rugosité et les défauts d'une optique. L'AFM utilise une sonde de balayage pour mesurer le profil de surface à une fréquence spatiale très élevée. C'est une mesure qui prend beaucoup de temps, sa plus grande valeur réside donc dans la tâche essentielle de validation et de surveillance du processus, où une fraction de la surface est échantillonnée pour fournir une représentation statistiquement significative de la performance du processus.

La spectroscopie à cavité optique est un autre outil important de vérification des traitements optiques de haute qualité. Dans une mesure à cavité optique, la lumière est réfléchie ou transmise plusieurs fois par un élément optique. Chaque fois que la lumière interagit avec cet élément optique, elle subit les pertes qui se produisent au niveau de ces surfaces. Cette mesure est très sensible à la performance du traitement, et toute aussi sensible à la qualité de la finition de la surface. Comme cette mesure est basée sur un temps de décroissance, elle peut être effectuée avec une très grande précision.

L'AFM et la spectroscopie à cavité optique sont deux méthodes de métrologie qui peuvent donner confiance dans les capacités d'un fournisseur de composants. Les fabricants d'optiques expérimentés comprendront également les nuances de l'examen visuel, à l'œil nu ou à l'aide d'un microscope et à l'échelle microscopique. Une métrologie compétente est essentielle lorsqu'il s'agit de traiter des pics de puissance élevés communs aux systèmes laser, en particulier pour les systèmes laser dans la région spectrale UV à haute énergie. II ne s'agit pas seulement d'une question de performance du système, mais aussi d'une question de durée de vie du système.

\section{LIDT - CE QUE VOUS NE CONNAISSEZ PAS VOUS FAIT DU TORT}

Le seuil de dommage laser (LIDT) est une spécification qui quantifie le pic de puissance ou d'énergie maximal qu'un élément optique peut tolérer avant de s'endommager. En général, les dommages laser se produisent lorsque l'énergie est absorbée ou concentrée au niveau d'une imperfection dans un élément optique. L'énergie supplémentaire absorbée entraîne un échauffement au niveau du défaut, qui à son tour entraîne une absorption plus importante et un échauffement conséquent, ce qui provoque des dommages permanents à la surface optique. Diverses imperfections peuvent agir comme des sites de dommages laser, y compris les rayures et les creux, c'est pourquoi la finition de la surface est particulièrement importante pour les optiques laser. Les imperfections qui causent des dommages ne sont pas toujours détectables, car les dommages laser peuvent être dus à des défauts, des résidus ou des dépôts à l'échelle du nanomètre.

Ces dernières années, on pensait que le LIDT était principalement dû à une exposition unique à une énergie dépassant une seule valeur seuil, qui pouvait être déterminée pour une conception donnée. Aujourd'hui, il est reconnu qu'il s'agit d'un phénomène complexe dans lequel l'exposition cumulative à des niveaux d'énergie inférieurs, ou peut-être à de courts pics dus à des fluctuations statistiques de puissance dans un faisceau d'énergie inférieure, peut contribuer à une dégradation qui finit par entraîner des dommages permanents. Cela signifie que plus d'une valeur pourrait être nécessaire pour quantifier le niveau d'exposition sûr aux rayonnements laser, et des travaux sont en cours pour fournir une définition plus complète des paramètres appropriés pour le LIDT. Une fois de plus, ce problème est exacerbé aux longueurs d'onde courtes et de haute énergie, car l'exposition aux UV affecte directement la structure des liaisons chimiques.

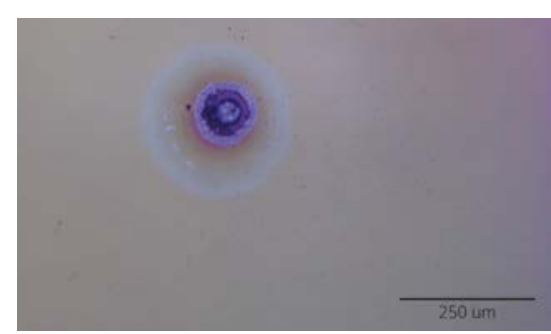

Figure 2. Défaut de traitement causé par un laser UV. Ce type de dommages peut entraîner une importante baisse des performances.

Les concepteurs de tout système optique de précision doivent spécifier avec soin les composants optiques pour atteindre les performances souhaitées du système. Une spécification appropriée devient encore plus importante dans les systèmes laser, où les concepteurs doivent accorder une attention particulière à la qualité de surface. Les concepteurs travaillant avec des systèmes UV ont une tâche encore plus difficile, car le rayonnement UV diffuse plus et transporte plus d'énergie que ses homologues visibles et infrarouges de plus grande longueur d'onde. Il est important non seulement de spécifier correctement les composants, mais aussi de s'assurer que leur fournisseur peut effectuer les mesures selon les spécifications indiquées.

Même avec tous ces défis, les concepteurs de systèmes peuvent être rassurés par le fait que la technologie de fabrication et de métrologie continue de s'améliorer et que les fournisseurs qui mêlent les nouvelles technologies à leur expertise éprouvée seront bien placés pour répondre aux spécifications les plus exigeantes. Les concepteurs doivent entamer très tôt une conversation avec leurs fournisseurs potentiels pour s'assurer que tous les facteurs importants sont pris en compte. Le fabriquant doit être en mesure de répondre aux questions sur les spécifications et la métrologie avant un achat: les concepteurs doivent donc rechercher un fournisseur expérimenté sachant effectuer les mesures pertinentes et discuter de la manière dont son expertise peut répondre aux besoins des concepteurs.

CONTACT: S. Vandendriessche, Laser Optics Product Line Manager - Cory Boone, Technical Marketing Engineer, Edmund Optics. For more info, please contact techsup@edmundoptics.eu. 